Cuestiones de sociología

ISSN: 2346-8904

publicaciones@fahce.unlp.edu.ar

Universidad Nacional de La Plata

Argentina

\title{
Notas sobre la gestación de una sociología de la atención médica de embarazos y partos
}

\author{
Castrillo, Belén \\ Notas sobre la gestación de una sociología de la atención médica de embarazos y partos \\ Cuestiones de sociología, núm. 22, 2020 \\ Universidad Nacional de La Plata, Argentina \\ DOI: https://doi.org/10.24215/23468904e093
}

Atribución no comercial compartir igual (CC BY-NC-SA) 4.0 
Notas de Investigación

\section{Notas sobre la gestación de una sociología de la atención médica de embarazos y partos}

Notes on the gestation of a sociology of medical care for pregnancies and births

Belén Castrillo

DOI: https://doi.org/10.24215/23468904e093

UNLP/CONICET, Argentina

mbcastrillo@gmail.com

\section{Resumen:}

La presente es una nota de investigación que reconstruye algunos de los resultados de una tesis doctoral en ciencias sociales sobre atención médica de embarazos y partos en la ciudad de La Plata entre 2013 y 2019. En particular, se repone una lectura de los resultados en tres niveles: a nivel macro (los procesos sociales estructurales que atraviesan los modelos de atención obstétrica), a nivel meso (las prerrogativas institucionales tensionando los derechos de las usuarias) y a nivel micro (las experiencias de mujeresmadres, varones-padres y profesionales de la salud sobre la atención de nacimientos para el caso de estudio).

Palabras ClaVe: Atención obstétrica, Tesis doctoral, Sociología de la salud.

\section{Abstract:}

In what follows, I replenish some elements of the doctoral research from a reading at three levels of the results: at the macro level (structural social processes), at the meso level (the institutional prerogatives stressing) and at the micro level (the experiences of women-mothers, boys-fathers and health professionals about birth care in La Plata for the study period).

KEYWORDS: Obstetric care, Doctoral thesis, Health sociology.

\section{LA PREGUNTA POR CÓMO NACEMOS}

Unos días después de dar a luz o cuando asoma la panza, en alguna plaza o reunión familiar, las mujeres gestantes y las puérperas reciben con naturalidad la pregunta “¿quién te hace/hizo el parto?”. En esta inquietud, y en su respuesta que incluye un apellido, se cristalizan sentidos y representaciones dominantes sobre cómo son y deberían ser abordados, atravesados y atendidos los procesos de embarazo y parto (PEP). Se trata de lo sedimentado sobre quiénes son lxs protagonistas del hecho, quién decide, cuáles son los modos socialmente legitimados de atenderlos y vivenciarlos, entre otras cuestiones. Hacer partos es un aspecto que subyace al universo de la atención de los PEP y tiene una gran carga simbólica a la hora de representar la labor profesional, en tanto la nominación de la tarea obstétrica emerge como hacer los partos. Y entonces allí caben las preguntas: ¿no son las mujeres gestantes \#y los fetos\# quienes hacen los partos? ¿Es lo mismo hacer el parto que parir? La normalización de este concepto que encierra en sí mismo una definición del quehacer profesional deja por fuera todo tipo de posibilidad de un rol protagónico de la mujer gestante en su propio proceso reproductivo. Asimismo, la acción de hacer el parto conlleva una visión intervencionista del suceso perinatal, en el cual no existe la posibilidad de estar expectante, acompañar, asistir, porque parecer ser que, tal como expresa Erviti (2010), lxs profesionales se presentan como "actores obligados a intervenir", responsables de evaluar el riesgo y "de definir las acciones" (p. 10).

La tesis doctoral ${ }^{1}$ a la que se refiere esta nota reconstruye y problematiza esta disputa sobre modelos de atención obstétrica que esquematicé en torno a hacer partos y parir. Básicamente, la tesis reconstruye una disputa principal que recoge actorxs, posiciones asimétricas de poder, prácticas y representaciones, entre dos modelos de atención médica de PEP: el intervencionista/medicalizado (bacer partos) y el humanizado/ respetado (parir). A lo largo de los capítulos, voy mostrando cómo la construcción de estos dos modelos 
(no sólo analítica sino, principalmente, como categorías del campo) articula diversas tensiones y ante la cual propongo pensar los grises entre los dos polos. Si puede decirse que estamos en una época de transición entre los dos modelos o de crisis del primero (impulsada desde el Estado, organizaciones de la sociedad civil, activismo feminista y algunos movimientos médicos), fue relevante también reponer las resistencias, muchas veces propugnadas por las parturientas, sujetas a una socialización médica tradicional que reproduce el modelo intervencionista.

En lo concreto, a lo largo de seis años, me dediqué a construir como problema sociológico el estudio de la atención médica de embarazos y partos, con el propósito de comprender la naturalización, extensión e invisibilización de la violencia obstétrica. A través de una extensa revisión bibliográfica de artículos y de tesis de las ciencias sociales sobre la temática, establecí áreas de vacancia, categorías, referentes conceptuales y experiencias diversas que sirvieron de guía y marco interpretativo de los datos producidos en el trabajo de campo. La instancia empírica de investigación se desarrolló principalmente entre 2015 y 2019 y fue abordada desde un enfoque cualitativo flexible y con un criterio de heterogeneidad y saturación. A través de un diseño muestral intencional por bola de nieve, implicó cuatro áreas de inserción: a) entrevistas a jefas de tocoginecología de las maternidades públicas de la ciudad de La Plata y a funcionarias del área de Maternidad e Infancia del Ministerio de Salud provincial (2015); b) entrevistas a profesionales de la salud obstétricas que desarrollan su quehacer profesional en ámbito público, privado y domiciliario (2015-2018); c) entrevistas a parejas de mujeres-madres y varones-padres que atendieron sus PEP en el sector público, privado y domiciliario (2015-2019); y d) observación con distintos grados de participación en 30 encuentros de seis cursos de preparto de la ciudad (2016-2017), de instituciones públicas, privadas y grupos que asisten partos en domicilio.

Se trató de una investigación que contó con el financiamiento de tres entidades de ciencia y técnica (CIC, UNLP y CONICET) ${ }^{2}$ y que se desarrolló en paralelo a la conformación y consolidación de un equipo de investigación sobre representaciones y prácticas en torno al proceso salud/enfermedad/atención con perspectiva de género en la facultad. ${ }^{3}$

En lo que sigue, repongo algunos elementos de la investigación doctoral a partir de una lectura en tres niveles de los resultados: a nivel macro (procesos sociales estructurales), a nivel meso (las prerrogativas institucionales tensionando derechos de las usuarias) y a nivel micro (las experiencias de mujeres-madres, varones-padres y profesionales de la salud sobre la atención de nacimientos en La Plata para el período de estudio).

\section{LO MACRO: PROCESOS SOCIOESTRUCTURALES}

El escenario actual respecto a la atención obstétrica se puede describir así: dos leyes de avanzada, ${ }^{4}$ el reconocimiento estatal e internacional de la problemática, la evidencia científica disponible para hacer un llamado urgente a desmedicalizar y desintervenir los nacimientos, y de todos modos la cesárea convertida en epidemia, el parto construido como un trauma y la idea de que quien hace los partos es el/la médicx y no la mujer y el/la recién nacidx. El propio proceso de deconstrucción me llevó a preguntarme cómo había sido posible que se naturalizara, internalizara y deseara parir con vías, hormonas sintéticas, maniobras externas, sin el mínimo poder de decisión sobre cómo, dónde, con quién. La violencia obstétrica, entonces, es el trasfondo de esta investigación en la que propongo pensar que existen tensiones y disputas en su definición: esto es, se trata de una problemática social en la que hay una tensión entre las nominaciones objetivas y externas y las apropiaciones subjetivas, la autoidentificación como víctimas y la posibilidad de cuestionar la autoridad médica y su definición, cimentada histórica y socioculturalmente, de cómo es nacer y parir en nuestra sociedad. 
En este marco, la indagación conceptual llevó a considerar la incidencia de ciertos procesos socioestructurales como la tensión entre el pretendido enfoque de derechos humanos y la perspectiva de género con una atención basada en una formación médica intervencionista, androcéntrica y patriarcal, con preeminencia del enfoque de riesgo y una profunda medicalización y patologización del proceso perinatal (Camacaro Cuevas, 2000, 2008a, 2008b y 2009; Camacaro Gómez, 2009; García Jordá et al, 2012; Mitjavila, 1998; Gainza et al, 2013; Sadler, 2003). Lo que está por detrás y articula este enfoque es la perspectiva sobre la doble dimensión de la práctica médica: como acción técnica y como acción moral o estructura de dominación (Schraiber, 2010; Castro y Erviti, 2015). El enfoque teórico-epistemológico al que subscribo privilegia la intersección de género y salud para analizar la conceptualización y atención médica de los procesos reproductivos (Esteban, 2001; Vals Llobet, 2009; Castañeda Abascal, 2014; De Lauretis, 1996).

Las tensiones macrosociales son construidas analíticamente en la tesis a partir de dos modelos, el intervencionista y el humanizado, con bases en las relaciones de poder, la tensión entre normativas, protocolos de acción profesional, establecimiento de derechos humanos y la revisión de prácticas médicas ${ }^{5}$ para limitar el sobreintervencionismo obstétrico. La construcción analítica de modelos me permitió confrontar las experiencias reales (institucionales y personales) y problematizar los distintos modos de concebir y atender los nacimientos.

FIGURA 1

Rasgos centrales de los modelos analíticos de atención obstétrica.

\begin{tabular}{|c|c|}
\hline ATENCIŌN OBSTĖTRICA HEGEMÖNICA & ATENCIŌN OBSTÉTRICA HUMANIZADA \\
\hline$>>$ HEGEMONIA MEEDICA $\ll$ & >>ENFOQUE FISIOLOGICOく< \\
\hline$>$ SABER EXPERTO $<<$ & $\gg$ DEMOCRATIZACIOON DEL SABER MÉDICO\& \\
\hline$\Rightarrow$ MEDICALIZACIÓN Y PATOLOGIZACIÖN $<<$ & >CCENTRALIDAD DEL SABER FEMENINO E \\
\hline $\begin{array}{l}>>\text { ENFOQUE DE RIESGO REPRODUCTIVO } \\
>\text { TECNOLOGIA, PRODUCCION Y TIEMPOS } \ll<\end{array}$ & >CRITERIO MÉDICO: NECESIDAD Y \\
\hline$\gg$ MECANISMOS DE DOMINACIONNY & PARTICULARIDAD $<<$ \\
\hline DESAUTORIZACIŌN $<<$ & $\begin{array}{c}\text { > } \\
\text { ENFOQUE DE DERECHOS HUMANOS Y } \\
\text { GENERO }\end{array}$ \\
\hline
\end{tabular}

Elaboración propia

La construcción teórico-metodológica de estos modelos a partir de los antecedentes teóricos y el entramado normativo, que constituyen el nivel macro del estudio, fue contrastada con las instituciones concretas y las experiencias de los sujetos de estudio, para encontrar los grises y los puentes entre los extremos. El aporte de la tesis radica, justamente, en complejizar el estudio al describir las convivencias contradictorias de estos rasgos en la atención concreta.

\section{LO MESO: INSTITUCIONES SANITARIAS Y MÉDICAS}

La reacción al primer modelo, y la construcción del segundo, se ampara en la propuesta estatal de una política de humanización de la asistencia al parto que combina dos dimensiones centrales: la garantía de los derechos sexuales y reproductivos de las mujeres, y, por ende, de la ciudadanía y autonomía de las mujeres con el derecho a decidir sobre su propio cuerpo, y la crítica-transformación de las prácticas médicas obstétricas para apuntar al cumplimiento de esos derechos. Los lineamientos de esta política se establecen en guías ministeriales y diversos protocolos. ${ }^{6}$ En líneas generales, se puede afirmar que la letra de estas guías que referencian la política de humanización expresa visiones progresistas que tensionan la realidad en la que se atiende hoy. Sin embargo, proponen un horizonte que no ve constricciones estructurales del sistema sanitario y hospitalario y de la profesión médico-obstétrica general, y desatiende disputas, (des)acuerdos, condiciones y posibilidades de lxs sujetxs, colectivos e instituciones implicados; al tiempo que se presenta como una alternativa de cambio, 
reevaluación y redefinición de prácticas médicas con el fin de garantizar derechos. En el análisis, me centro en problematizar estas guías en tres ejes: primero, el enfoque de riesgo reproductivo utilizado para ponderar los partos; segundo, la cuestión de las prácticas médicas aconsejadas y desaconsejadas para la intervención en estos casos; y, tercero, el foco en los derechos sexuales y reproductivos de las mujeres que buscan garantizarse en este período del proceso perinatal.

La descripción y abordaje de esta política de humanización es la excusa para comenzar a analizar el caso de estudio: la atención obstétrica en la ciudad de La Plata. Para ello me centro en conocer cuáles son las condiciones macro de la atención y la brecha con esta tendencia humanizadora, a partir del análisis de lo que sucede en la atención del sector público. A partir de datos duros y entrevistas con funcionarias y jefas de servicio de tocoginecología de tres de las cuatro maternidades públicas de la ciudad de La Plata, en la tesis reconstruyo los pormenores de esta brecha entre lo que debería ser nacer actualmente y lo que efectivamente sucede: alta predominancia del intervencionismo médico, visible en altas tasas de cesáreas, inducciones y episiotomías, amparado en una hegemonía del enfoque de riesgo obstétrico aceptado acríticamente por médicxs y usuarixs.

En particular, analizo la vulneración de derechos humanos de las mujeres y sus familias en la atención perinatal (establecidos por la ley de parto humanizado vigente desde 2004) y encuentro que la posibilidad de ser acompañadas por la persona que ellas deseen, la libre elección de la posición para parir y la no separación madre-recién nacidx, entre otros, no están garantizados en la atención pública. Asimismo, analizo algunos datos estadísticos que permiten confirmar que la tendencia obstétrica intervencionista sigue siendo la hegemónica:

\section{GRÁFICO 1}

Índice de cesáreas en las cuatro maternidades públicas de la ciudad de La Plata (2013-2017)

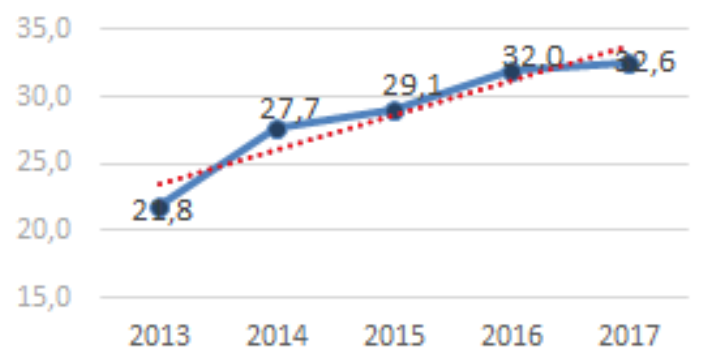

Elaboración propia sobre la base de SIP PBA 2014-20187

Observando el gráfico, se nota cómo aumentó el índice de cesáreas en relación con los nacimientos en el sector público en La Plata para el período señalado. ${ }^{8}$ En efecto, en 2017, el 32,6\% del total de los nacimientos registrados fueron por cesárea (sobrepasando el límite ideal que propone la OMS de 10-15\% de cesáreas). El dato sustantivo es cómo aumentó este índice: en 2017 se produjeron 49,5\% de nacimientos por cesárea más que en 2013. La nota de color es que en 2015, tras once años de su sanción, se reglamentó la ley de parto humanizado, lo que lleva a preguntarnos qué incidencia tienen las leyes en la transformación real de la atención obstétrica.

\section{LO MICRO: EXPERIENCIAS PROFESIONALES Y PERSONALES}

Finalmente, la tesis reconstruye experiencias subjetivas en dos sentidos: las que reflejan el quehacer profesional de médicxs, parteras, neonatólogxs y otros miembros del equipo de salud, y las que enuncian las vivencias de atención obstétrica de mujeres-madres y varones-padres. 
A partir de las primeras, logro reconstruir el campo profesional obstétrico como un campo de disputa entre los dos modelos: el hacer partos (intervencionista) y "la otra obstetricia", ligada al paradigma humanizador. A partir de la creación de un dispositivo indagador sobre los primeros y los últimos partos en los que participaron como profesionales de la salud, fue posible advertir cómo, siguiendo a Castro (2014), se va constituyendo el habitus médico autoritario alrededor de la atención obstétrica, vinculado al modelo intervencionista. De igual modo, emergió cómo ciertos giros y quiebres en la trayectoria asistencial viraron su modo de trabajo hacia paradigmas fisiológicos y de respeto a los derechos y necesidades de las mujeres.

En relación con las experiencias de las parejas de madres/padres, a través del análisis de la atención médica de todo el PEP fue posible ver cómo se van tensionando los modelos en los distintos ámbitos de atención (público, privado y domicilio) y cómo se va dando el proceso social de construcción y cuestionamiento a los sentidos culturales dominantes sobre cómo debería ser/es parir y nacer.

\section{Cierre: Grises y Puentes}

A través de la presente nota, se describieron algunas de las principales líneas de análisis que la tesis doctoral sobre la atención médica de PEP en la ciudad de La Plata que desarrollé me permitió abordar. A partir de la construcción de modelos típico-ideales como herramienta heurística de conocimiento, fue posible problematizar las experiencias concretas a distintos niveles y advertir cómo son más comunes los grises y puentes en la heterogeneidad real que las respuestas ortodoxas a uno u otro modelo. Y cómo, desde una perspectiva de derechos humanos y género, se puede gestar una sociología que se pregunte por cómo parimos y nacemos.

La propuesta de la tesis ha sido construir saberes situados (Femenias y Soza Rossi, 2011) sobre cómo se nace y se pare en la actualidad y cómo los sujetos experimentan la atención médica de dichos PEP. Mientras escribía esta tesis (2013-2019), nacieron casi 80.000 personas en la ciudad de La Plata. Es posible decir que la mayoría de esos nacimientos se dieron en el marco de un modelo asistencial intervencionista, en el que primaron los criterios y necesidades médicas, que tienden a patologizar el proceso, sobre los derechos y deseos de las mujeres y sus familias. La apuesta es porque cada vez más el porcentaje de nacimientos placenteros, respetuosos y fisiológicos crezca.

\section{ReFERENCIAS}

Camacaro Cuevas, M. (2000). Experiencia del parto. Proceso de la mujer o acto médico. Carabobo: Universidad de Carabobo.

CamacaroCuevas, M. (2008a). La construcción discursiva médico-obstétrica en el proceso reproductivo de las mujeres. Revista Ensayo y error, Año XVII, No 35. 95-115.

Camacaro Cuevas, M. (2008b). Dos sentencias masculinas: parirás con dolor... parirás acostada. En: Delgado de Smith, Y. D. y González, M. C. (Comps.) Mujeres en el mundo. Migración, género, trabajo, historia, arte y política. Valencia (Venezuela): Laboratorio de investigación en estudios del trabajo (LAINET). Facultad de Ciencias Económicas y Sociales. Carabobo: Universidad de Carabobo. 293-310.

Camacaro Cuevas, M. (2009). Patologizando lo natural, naturalizando lo patológico? improntas de la praxis obstétrica. Revista venezolana de estudios de la mujer, 14(32), 147-162.

Camacaro Gómez, M. (2009). Histerectomía: una práctica médica entre la profilaxis y la castración. En Delgado de Smith, Y.D. y González, M.C. (Coords.) Mujeres en el mundo: ciencia, género, migraciones, arte, lenguaje y familia. Valencia (Venezuela): Laboratorio de investigación en estudios del trabajo (LAINET). Facultad de Ciencias Económicas y Sociales. Carabobo: Universidad de Carabobo. 217-232.

Castañeda Abacal, I. (2014). El marco teórico en la investigación en salud con enfoque de género. Revista Cubana de Salud Pública. 40(2), 249-257. 
Castro, R. (2014). Génesis y práctica del habitus médico autoritario en México. Revista Mexicana de Sociología, abriljunio, 76(2), 167-197.

Castro, R. y Erviti, J. (2015). Sociología de la práctica médica autoritaria. Violencia obstétrica, anticoncepción inducida $y$ derechos reproductivos. México: UNAM-CRiM.

De Lauretis, T. (1996). La tecnología del género. Mora, 2, 6-34.

Erviti, J. (2010). "Construcción de los objetos profesionales, orden corporal y desigualdad social. Una reflexión en torno a las interacciones médicos-usuarias de servicios ginecológicos”. En R. Castro, y A. López Gómez(Eds.). Poder médico y ciudadania: el conflicto social de los profesionales de la salud con los derechos reproductivos en América Latina: avances y desafíos en la investigación regional. Montevideo-México: Universidad de la República-UNAM, CRIM. 101-122.

Esteban, Mari Luz. (2001).El género como categoría analítica. Revisiones y aplicaciones a la salud. Perspectivas de género en salud. Fundamentos cientificos y socioprofesionales de diferencias sexuales no previstas, p. 25-51.

Femenías, M. L. y Sosa Rossi, P. S. (2011). Saberes situados, teorias trashumantes. La Plata: UNLP-Facultad de Humanidades y Ciencias de la Educación- CINIG-IdHCS-CONICET.

Gainza, P., Labastie, M., \& Magnone-Alemán, N. (2013). La atención contemporánea del parto: jaque a la perspectiva de derechos humanos. Uluá Revista de Historia, Sociedad y Cultura, 22, 175-96.

García Jordá, D. y Díaz Bernal, Z. (2010). Perspectiva antropológica y de género en el análisis de la atención al embarazo, parto y puerperio. Revista cubana de salud pública, 36, 330-336.

Mitjavila, M. (1998). El saber médico y la medicalización del espacio social. Documento de Trabajo DTS. Montevideo: Facultad de Ciencias Sociales.

Sadler, M. (2003). Asi me nacieron mi hija. Aportes antropológicos para el análisis de la atención biomédica del parto hospitalario (Tesis de grado). Santiago de Chile: Universidad de Chile.

Schraiber, L. (2010) "Acción técnica y acción moral: el doble carácter de la práctica de los profesionales de la salud. Apuntes para el debate." Poder médico y ciudadania: el conflicto social de los profesionales de la salud con los derechos reproductivos en América Latina. Montevideo: Universidad de la República. 11-26.

Valls Llobet, C. (2009). Mujeres, salud y poder. Madrid: Cátedra Ediciones.

\section{Notas}

1 "Hacer partos y parir: hacia una sociología de la atención médica de embarazos y partos. Experiencias de mujeres-madres, varones-padres y profesionales de la salud obstétrica de La Plata (2013-2019)”. Tesis para optar al grado de Doctora en Ciencias Sociales de la FaHCE/UNLP, en la que fui dirigida por la Dra. Licia Pagnamento y que defendí en julio de 2019.

2 Obtuve diversas becas durante el desarrollo de la investigación: Beca de perfeccionamiento CIC/PBA (2014-2015), Beca tipo A UNLP (2015-2018) y Beca de Finalización de Doctorado de CONICET (2018-2020).

3 Se trata de un equipo (del que participé en su fundación) que desde 2014 ha ido desarrollando proyectos de I+D en torno al estudio sociológico de los procesos de salud/enfermedad/atención con perspectiva de género, dirigido por mi directora de tesis, la Dra. Licia Pagnamento.

4 Ley 26.485, "de Protección Integral para Prevenir, Sancionar y Erradicar la Violencia contra las Mujeres en los ámbitos en que desarrollen sus relaciones interpersonales", de 2009, conocida como "ley de violencia de género", que nomina la violencia obstétrica como una de las seis modalidades de violencia contra las mujeres. Ley 25.929 (sancionada en 2004 y reglamentada en 2015), conocida como "ley de parto humanizado", que establece los derechos de las mujeres y sus familias durante el proceso de atención perinatal.

5 En particular, desarrollo el índice "10-10-10" propuesto por la OMS, que sostiene que las cesáreas, las inducciones y las episiotomías no deberían superar esos porcentajes en la atención obstétrica.

6 Guía 1: "Maternidad segura y centrada en la familia con enfoque intercultural: conceptualización e implementación del modelo" (2011, Ministerio de Salud de Nación); Guía 2: "Guía para la atención del parto normal en maternidades centradas en la familia” (2012, Ministerio de Salud de Nación); Guía 3: "Guía de procedimientos para el control del embarazo y la atención del parto y puerperio de bajo riesgo” (2012, Ministerio de Salud Provincia Buenos Aires).

7 Agradezco especialmente a la Lic. Jimena Coniglio por ayudarme a construir e interpretar este cuadro y los valores resultantes. 
Cuestiones de SOciología, 2020, NúM. 22, ENERo-Diciembre, ISSN: 2346-8904

8 Este período está determinado por la disponibilidad y accesibilidad a datos oficiales.

CC BY-NC-SA 